\title{
The Dynamic Impacts of the Global Shipping Market under the Background of Oil Price Fluctuations and Emergencies
}

\author{
Zihan Chen, ${ }^{1}$ Xiaokong Zhang $\mathbb{D}^{2},{ }^{2}$ and Jian Chai ${ }^{2}{ }^{2}$ \\ ${ }^{1}$ Accounting School, Henan University of Economics and Law, Zhengzhou 450046, China \\ ${ }^{2}$ School of Economics and Management, Xidian University, Xi'an 710126, China \\ Correspondence should be addressed to Xiaokong Zhang; xkzhang_1996@stu.xidian.edu.cn and Jian Chai; chaijian0376@126.com
}

Received 9 September 2020; Revised 13 December 2020; Accepted 30 December 2020; Published 11 February 2021

Academic Editor: Xueyong Liu

Copyright (c) 2021 Zihan Chen et al. This is an open access article distributed under the Creative Commons Attribution License, which permits unrestricted use, distribution, and reproduction in any medium, provided the original work is properly cited.

With growing uncertainty about the evolution of the global landscape, it is of great practical significance to explore the nonlinear dynamic adjustment relationship among the world oil market, the global bulk shipping market, the stock market, and economic growth in China. This paper applied the TVP-SV-VAR model and selected quarterly data from 1998 to 2020 to explore the dynamics. The results indicated that the impact intensity of BDI on China's economy had a "positive" to "negative" change in different lag periods. This was mainly due to the fact that the negative impact of higher freight prices on China's economy outweighed the positive impact of higher trade volumes on China's economy. The impact intensity of BDI on GDP had a distinct medium- to long-term effect. A positive BDI shock had a dampening effect on stock prices in the short and medium term, while a positive BDI shock could promote stock market prosperity in the long-term perspective. The impulse responses of SSE and GDP to BDI showed that the external shipping market shocks to China's stock market and economic growth gradually became smaller over time. For impulse response at three different time points, the impact intensity of the BDI to GDP varied at different time points, with the largest shock during the financial crisis in 2008, followed by the shock during the oil price crash in 2014, and the smallest during the COVID-19 epidemic. This demonstrated that the external shipping market's influence on Chinese economic growth and stock market has gradually weakened over time, illustrating the enhancement of Chinese risk-resilience capacity.

\section{Introduction}

With the further advance of economic globalization and the rapid development of international trade, the scope and degree of international trade markets increasingly have extended and strengthened. Meanwhile, the surging demand for maritime trade derived from the international trade market has made the global shipping market increasingly prominent. Dry bulk transport, including coal, iron ore, and grain, is the mainstay of the international shipping industry, which is related to the basic survival demands of nations, the basic material guarantee for the survival of the countries, and the essential condition for maintaining the standard of living desired by the nationals. Therefore, in a certain sense, compared with container transport, general cargo transport, and liquid bulk transport, the dry bulk market, which is responsible for the transportation of major raw materials and materials, plays an increasingly crucial role in the international shipping market and international trade. The Baltic Dry Index (BDI) is an international authoritative index for measuring international shipping conditions, the barometer and wind vane of the international shipping market, the leading index reflecting international trade conditions, and the microcosm of the global economy. In 2008, when the BDI reached a record high, a large number of investors were attracted to the maritime industry. The financial crisis broke the gold rush and shipping rates plummeted. The slump and depression in the shipping industry had continued until 2013, when the shipping market was rejuvenated, and the BDI returned to 2000. However, in 2014, the Ebola virus ravaged the whole of Africa. The Danish Poyan Oil Company applied to the court for bankruptcy, the supply of ships and bunker oil was seriously affected, and the BDI began to decline precipitously 
in the market downturn. The shipping market then went into a slow recovery, yet its upward trend was dampened by the sudden onset of COVID-19. The main factors influencing the fluctuation of the BDI include global GDP growth rate, global demand for iron ore and coal transportation, global demand for grain transportation, global supply of ship tonnage, average international bunker price, war, and natural disasters. Among them, fuel oil prices, economic, climate, and unexpected events have a more profound impact on BDI. Firstly, fuel costs can account for $40 \%$ of a ship's operating costs. Drastic changes in oil prices may cause a certain degree of disturbance to the BDI. Secondly, from the trend of BDI over the years, the bulk freight rate is determined by the relationship between supply and demand, but the concentration of dry bulk transport industry is low, and the change of the supply side (effective capacity) is relatively slow. Therefore, the short-term fluctuation of the BDI is mainly dominated by the demand side (transport demand), which is greatly influenced by the change of economic prosperity. In terms of the major regions connected by BDI routes, key routes from China and the United States are included. In recent years, China, as a major demander of bulk dry bulk cargoes, has seen a growing demand for imports of bulk raw materials, most notably iron ore and coal, which rank among the highest in terms of seaborne imports. Thus, as a bellwether of trade data for trade-active countries, the BDI is closely related to the trade volume and GDP growth of China and the US and, even more so, reflects the degree of economic prosperity in China. In addition, other contingencies at the supply and demand level can also phase in freight rates, such as the financial crisis and COVID-19.

As a result, the shipping market is dependent on the oil market and on international trade. As the world's largest trading country, the interaction between the oil market and shipping market has a profound impact on investor sentiment and trade volume, ultimately exacerbating China's macroeconomic fluctuations. Especially, in recent years, against the backdrop of structural changes in internal supply and demand, sharp fluctuations in oil prices and frequent unexpected events, the BDI fluctuates frequently and its influence on China's economy should not be underestimated. The stock market acts as a macroeconomic barometer, and the macroeconomic functions profoundly affect the stock market volatility. Many scholars have confirmed the existence of a positive correlation between the BDI, the US stock market, and the US dollar index, but there are rare studies on the mechanism of the influence of the BDI and the Chinese stock market. Against the background of increasing economic uncertainty, it is of great practical significance to study the linkages among the world oil market, global shipping market, China's stock market, and economy. There have been numerous excellent reviews in the literature of the two-two relationship among the oil market, shipping market, stock market, and GDP, but studies which include the world oil market, global shipping market, Chinese stock market, and GDP in the same system are little investigated. Furthermore, there are only few studies that consider the nonlinear dynamic linkage between the variables. Thus, this paper attempts to use the TVP-SV-VAR model to analyze the nonlinear dynamic adjustment relationship among the world oil market, global shipping market, Chinese stock market, and economic growth and fully explore the inner influence mechanism between the variables. Only by identifying the potential shock effects of the world oil market and global shipping market, can we clarify the direction and path of policy adjustment based on the driving factors, thus effectively preventing the impact of oil prices and ocean freight rates, while reducing the possibility of unexpected shocks to Chinese economy and stock market. In addition, this paper examines the linkages and shock effects between markets under the major emergencies of the "financial crisis," "international oil price crash," and "COVID19 ," in order to provide useful scientific reference for industry insiders.

\section{Literature Review}

2.1. The Effect of the Oil Market on BDI, Stock Market, and Economic Growth. As a leading indicator of global shipping costs and a unit of measurement of commodity trading volume, the BDI was closely related to fluctuations in the world oil market. On the one hand, crude oil price was transmitted directly to the BDI through global shipping and transportation costs. On the other hand, changes in international market factors such as the global economic cycle, business cycle, and major emergencies would act on both the BDI and the international crude oil market at the same time, thus triggering a synchronized volatility trend in both markets [1]. There was a significant cross-correlation between the BDI and crude oil price, and the short-term multiple fractal characteristics were stronger than the longterm ones [2]. In accordance with this, Arigoni et al. [3] structured different scenarios of the effects of high and low BDI and oil prices and found same conclusions.

Oil price shocks have a significant impact on factor inputs in the production sector as well as on macroeconomic performance. In terms of macroeconomics, theoretical studies have shown that the positive shock of oil prices affects the input of relevant downstream production sectors through the cost channels of upstream raw materials and then affects the output [4-6]. In this process, imported inflation caused by oil prices directly affects the economy, triggering the reverse adjustment of the monetary and fiscal policies, which further indirectly affects the economic growth. As the largest oil importer, the Chinese economy cannot avoid the shock of international oil prices. Existing research studies have attempted to explore the response of Chinese economy to oil price shocks. Cheng et al. [7], based on a GARCH model, showed that uncertainty of oil price had a negative impact on China's real GDP. Zhao et al. [8] established a DSGE model to confirm that the influence of different types of oil price fluctuations had different approaches on economic growth, and rising oil prices driven by demand would produce long-term negative effects on China's GDP. Kim et al. [9] found that Chinese economy had a time-varying response to the oil price shock.

As far as the stock market was concerned, the causal relationship between the international crude oil market and 
the stock market largely depended on the development stage, energy structure, global crude oil market, and other factor endowments $[10,11]$. The existing research studies showed that crude oil market was the Granger cause of stock market volatility $[12,13]$. Phan et al. [14, 15] empirically demonstrated that the introduction of oil prices could significantly improve the accuracy of stock price forecasts. In addition, most studies showed that the two were preceded by a nonlinear time-lagged effect. Wen et al. [16] further suggested that oil price shocks had a negative effect in the short run but would turn positive in the long run. Ding et al. [17] used a SVAR model to prove that international crude oil price fluctuations had a significant Granger effect on investor sentiment in the Chinese stock market, and investor sentiment was contagious by international crude oil price fluctuations with an average lag of about 8 months.

2.2. The Effect of BDI on the Stock Market and Economic Growth. Numerous studies have analyzed the volatility spillovers and linkage mechanisms among the BDI, stock market, and economic growth. Existing research studies showed that the BDI had a long-term cointegration relationship with the US stock market Standard and Poor's Dow Jones Indices. Changes in the BDI could help explain the Dow Jones Indices [18]. Alizadeh and Muradoglu [19] further demonstrated that using the BDI for predicting other stock market fluctuations could significantly improve forecast performance. Besides, Lin et al. [20] confirmed that the BDI had a significant time-varying volatility spillover effects on commodity and stock markets by using the VARBEKK-GARCH-X model. Given the volatility spillover relationship among the BDI, commodity, and stock market, the BDI was closely related to global trade volatility; thus, the research related to the Baltic Dry Index (BDI) as a leading economic indicator was a hot issue in academic research [21-23]. A large number of scholars have favored the BDI as a leading indicator for predicting the macroeconomy $[24,25]$.

In summary, there has been a large amount of literature examining the two-two relationship among international oil markets, shipping markets, stock markets, and economic growth, providing evidence of their correlation and giving a factual and theoretical basis for the in-depth study of this paper. However, on the one hand, there are relatively few studies that include oil markets, stock markets, shipping markets, and GDP in the same system, and furthermore, there are only a few papers that consider the nonlinear dynamic linkages between the variables. On the other hand, the relationships among variables may present different results depending on the country, region, sample range, system changes, and methodological selection. Consequently, this paper employed the TVP-SV-VAR model to explore the nonlinear linkages between different markets and Chinese economic growth, which could theoretically enrich the current relevant studies and provided factual evidence for the nonlinear linkages between different markets and the Chinese economy in practice.

\section{Methodology}

Vector autoregression is a fundamental tool in econometric analysis and is widely used. However, the coefficient and variance in the traditional VAR model are constant. In many cases, the data generation process for economic variables appears to have randomly fluctuating drift coefficients. In addition, with constant changes such as institutional changes and policy preferences, large structural abrupt changes may occur in the economic system. At this time, the estimated time-varying coefficient of the VAR model may be biased because it ignores the possible changes of volatility in the disturbance. It is a great challenge to characterize and interpret the dynamic adjustment characteristics between observed variables, and thus, VAR is limited in use. In recent years, in order to capture the potential time-varying nature of the economic structure, random fluctuations are gradually incorporated into the TVP-VAR model. The TVP-SV-VAR model sets all parameters as a first-order random walk process, allows temporary and permanent deviations of parameters, and enables us to capture the possible timevarying properties of economic structure in a flexible and robust way, which can well describe the nonlinear and dynamic characteristics between variables. As a result, it is more advantageous at the practical application level.

The TVP-SV-VAR model is a variant of the traditional VAR model. The structural VAR model can be defined as follows:

$$
A y_{t}=F_{1} y_{t-1}+\cdots+F_{s} y_{t-s}+\mu_{t}, \quad t=s+1, \ldots, n,
$$

where $y_{t}$ is a $k \times 1$ vector of observed variables, $A, F_{1}, \ldots, F_{s}$ is a $k \times k$ matrix of coefficients, and $\mu_{t} \sim N(0, \Sigma \Sigma)$ can be described as a $k \times 1$ structural shock. Assuming that the coefficient matrix $A$ is a lower-triangular, the recursive method can be used to identify the structural relationship. Equation (1) can be transformed into the following form:

$$
y_{t}=X_{t} \beta_{t}+A_{t}^{-1} \Sigma_{t} \varepsilon_{t}, \varepsilon_{t} \sim N\left(0, I_{k}\right),
$$

where the parameter $\beta_{t}$, matrix $A_{t}$, and $\Sigma_{t}$ are all temporally variable parameters. It is assumed that the parameters to be evaluated follow the random walk process, which implies that temporary and permanent shifts in the coefficients are allowed. The drift coefficients can be used to capture possible nonlinearity, such as gradual or structural changes. The error term can be used to capture temporary shifts:

$$
\begin{aligned}
\beta_{t+1} & =\beta_{t}+\mu_{\beta_{t}}, \alpha_{t+1}=\alpha_{t}+\mu_{\alpha_{t}}, \\
h_{t+1} & =h_{t}+\mu_{h_{t}}, \\
{\left[\begin{array}{l}
\varepsilon_{t} \\
\mu_{\beta_{t}} \\
\mu_{\alpha_{t}} \\
\mu_{h_{t}}
\end{array}\right] } & \sim N\left[0\left[\begin{array}{cccc}
I_{k} & 0 & 0 & 0 \\
0 & \Sigma_{\beta} & 0 & 0 \\
0 & 0 & \Sigma_{\alpha} & 0 \\
0 & 0 & 0 & \Sigma_{h}
\end{array}\right]\right],
\end{aligned}
$$

where $\quad t=s+1, \ldots, n, \beta_{s+1} \sim N\left(\mu_{\beta_{0}}, \Sigma_{\beta_{0}}\right), \alpha_{s+1} \sim N\left(\mu_{\alpha_{0}}\right.$ ' $\left.\Sigma_{\alpha_{0}}\right)$, and $h_{s+1} \sim N\left(\mu_{h_{0}}, \Sigma_{h_{0}}\right)$. The model is a nonlinear statespace model considering the time variability of the 
perturbation term. Therefore, the maximum likelihood estimation requires repeated filtering for each set of parameters until the maximum value is reached, and the calculation is too large. This paper adopts the Bayesian method and MCMC simulation to effectively estimate the TVP-SV-VAR model so as to better depict the time-varying characteristics of coefficients and random volatility.

\section{Data and Preliminary Analysis}

4.1. Variable and Data. This paper focused on the nonlinear dynamic relationship among the world oil market, the global shipping market, the Chinese stock market, and GDP. The variables were selected as follows: (1) the selection of oil prices: firstly, Brent price and WTI price are important benchmark crude oil prices in the international oil market, and both futures markets have good price discovery functions. Numerous studies have shown that the price discovery function of the WTI crude oil futures market is more representative of the international benchmark crude oil price level [26-28]. With the development of the global economy, the advantages of Brent in terms of the scale of global spot trade and market liquidity are gradually revealed. Most of the findings have revealed that Brent plays an increasingly crucial role in the international crude oil pricing benchmark. Attanasi [29] proved that Brent futures market dominated in the international crude oil price discovery. In addition, Qingsong et al. [2] used multiple fractal trend mutual analysis to prove that the BDI and Brent had strong multiple fractal characteristics than WTI. Therefore, the Brent spot price was chosen as a proxy variable for the crude oil market in this paper; (2) the selection of stock price: the Shanghai Composite Index and the Shenzhen Component Index are two representative stock price indices in China. In terms of the range of constituent stocks, the constituent stocks of the Shanghai Composite Index are all stocks listed on the Shanghai Stock Exchange, reflecting the overall price movements of stocks listed on the Shanghai Stock Exchange. The Shenzhen Component Index is calculated by taking the stocks of 500 listed companies, mainly information technology companies, as the representative of the market. As a result, the Shanghai Composite Index is more representative than the Shenzhen Component Index. In terms of research on Chinese economic cycle and stock market, a large amount of literature has used the Shanghai Composite Index to represent the movement of stock prices [30,31]. This paper also chose the Shanghai Composite Index to represent the changes in stock prices in China. For the ease of description, the SSE was used instead of the Shanghai Composite Index; (3) the BDI index was adopted to represent changes in global shipping prices, and quarterly GDP was utilized as a proxy variable for Chinese economic growth.

For data processing, on the one hand, there were generally statistical problems such as too high lagged order and more parameters to be estimated when modeling using daily high-frequency data of BDI, Brent, and SSE, resulting in biased estimation results. On the other hand, since the GDP only had quarterly and annual data, in order to facilitate the analysis and ensure sufficient sample capacity, the mixed- frequency data were transformed into quarterly data with the same frequency. The data period was from the first quarter of 1992 to the second quarter of 2020, and the data were from the Wind database. In order to remove the effect of seasonal variation, all variables were seasonally adjusted using the X-12 technique. Next, a standardized treatment that removed the long-term trend and divided it by the standard deviation was applied to all variables in order to portray the short-term volatility of the variables and to remove the effects of the magnitudes.

\subsection{Preliminary Analysis}

4.2.1. Unit Root Test. The TVP-SV-VAR model requires the observed variables to be stationary, and in order to prevent the occurrence of pseudoregression, the unit root test should be performed on each variable before modeling. In this paper, ADF was used to check the stationarity of the variables. There are three main types, which include only the Intercept term, Intercept and trend term, and None. In general, as long as one type of unit root test passes (i.e., the null hypothesis is rejected), the variable is considered to be stationary. The results are shown in Table 1. The results showed that the BDI, Brent, and SSE rejected the null hypothesis at the $1 \%$ significance level, and GDP also passed tests for "Intercept," "Intercept and trend," and "None" at the $5 \%, 10 \%$, and $1 \%$ significance levels, respectively. In conclusion, all variables followed $I(0)$ and could be further analyzed empirically.

4.2.2. Parameter Test. This article used OxMetrics 6 software and the TVP-SV-VAR package written by Jouchi Nakajima, a measurement economist at the Bank for International Settlements, to estimate the model. According to the AIC, BIC, and SC criteria, the optimal lag for the TVP-SV-VAR was identified as 2 . Next, to compute the posterior estimates, we draw 10,000 samples using Monte Carlo Markov Chains (MCMC). The estimation results are shown in Table 2 and Figure 1. Table 2 provides the distribution of posteriors, the convergence diagnostics of Geweke, and inefficient factors computed by using MCMC sampling. For the convergence diagnosis, at the $5 \%$ significance level, the null hypothesis of convergence to the posterior distribution was rejected, implying that all parameters converge to the posterior distribution of the MCMC simulation, and we achieved the expected effect. Furthermore, the inefficient factors were rather low, within 100. The inefficient factor of $\left(\Sigma_{h}\right)_{2}$ was the largest, as high as 97.65 , which meant that there were only $102(10000 / 97.65)$ irrelevant samples in 10,000 simulations. That was why the number of efficient samples for parameters and state variables were sufficient. Figure 1 successively reveals sample autocorrelations, sample paths, and posterior densities of parameters for each row. The sample autocorrelations at the first row decreased rapidly and kept slightly ranging around the 0 level, illustrating that the majority of the sample was of low autocorrelation. The second row showed that the sample paths were stable without many 
TABLE 1: The results of unit root test.

\begin{tabular}{lcccccc}
\hline \multirow{2}{*}{ Variables } & \multicolumn{2}{c}{ Intercept } & \multicolumn{2}{c}{ Intercept and trend } & \multicolumn{2}{c}{ None } \\
& Statistics & $P$ values & Statistics & $P$ values & Statistics & $P$ values \\
\hline BDI & -4.5724 & $0.0003^{* * *}$ & -4.5421 & $0.0024^{* * *}$ & -4.6027 & $<0.0001^{* * *}$ \\
Brent & -4.4513 & $0.0005^{* * *}$ & -4.4115 & $0.0035^{* * *}$ & -4.4810 & $<0.0001^{* * *}$ \\
SSE & -5.5503 & $<0.0001^{* * *}$ & -5.5171 & $<0.0001^{* * *}$ & -5.5834 & $<0.0001^{* * *}$ \\
GDP & -3.2930 & $0.0185^{* * *}$ & -3.3143 & $0.0713^{*}$ & -3.2511 & $0.0014^{* * *}$ \\
\hline
\end{tabular}

***,**, and ${ }^{*}$ show the significance at the $1 \%, 5 \%$, and $10 \%$, respectively.

TABLE 2: The results of TVP-SV-VAR parameter estimation.

\begin{tabular}{lcccrc}
\hline Parameter & Mean & Std. dev. & $95 \%$ interval & Geweke & Inef. \\
\hline$\left(\Sigma_{\beta}\right)_{1}$ & 0.0229 & 0.0026 & {$[0.0184,0.0288]$} & 0.890 & 5.80 \\
$\left(\Sigma_{\beta}\right)_{2}$ & 0.0226 & 0.0026 & {$[0.0182,0.0284]$} & 0.692 & 0.822 \\
$\left(\Sigma_{\alpha}\right)_{1}$ & 0.0666 & 0.0208 & {$[0.0382,0.1184]$} & 0.071 \\
$\left(\Sigma_{h}\right)_{1}$ & 0.6182 & 0.1454 & {$[0.3684,0.9318]$} & 36.20 \\
$\left(\Sigma_{h}\right)_{2}$ & 0.9799 & 0.2776 & {$[0.5439,1.5799]$} & 0.053 & 30.74 \\
\hline
\end{tabular}

Note: $\Sigma_{\beta}$ and $\Sigma_{\alpha}$ are the values multiplied by 100 .
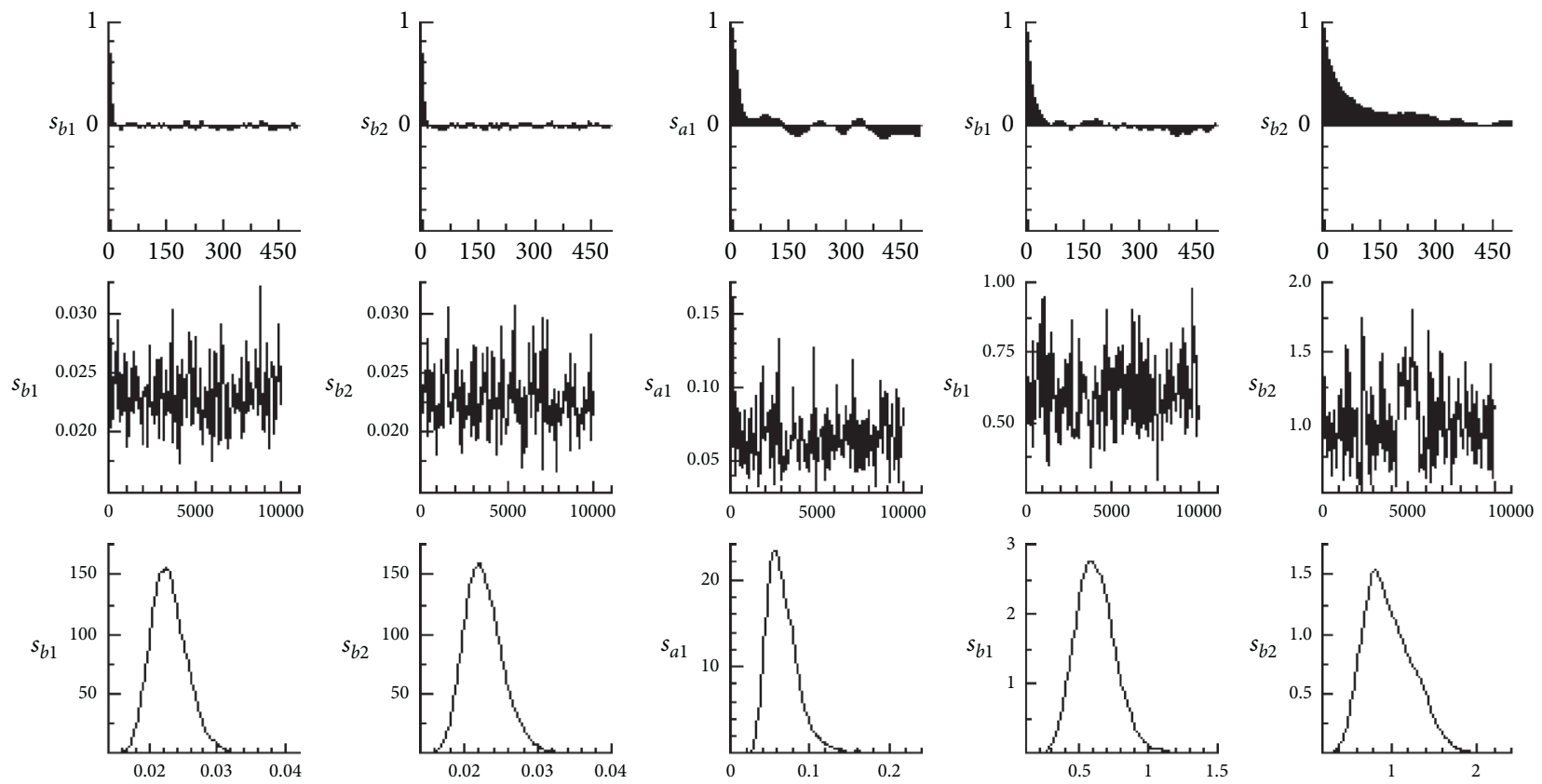

FIGURE 1: Sample autocorrelations (top), sample paths (middle), and posterior densities (bottom).

extreme values, which meant that the samples produced from MCMC were effective.

\section{Empirical Results}

This section mainly relied on two types of time-varying impulse response functions from the TVP-SV-VAR model to analyze the nonlinear dynamic interactions among the world oil market, the global shipping market, the Chinese stock market, and economic growth. The impulse response was sorted into the impulse response at different periods ahead and impulse response at different time points. Both impulse responses are influenced by the same factors and depict time-varying characteristics, but the impulse response is represented from a different perspective. Firstly, we chose shocks arising at 3 different periods ahead in terms of the response of variables at every time point: 2-quarter, 4quarter, and 8-quarter, portraying short-, medium-, and long-term phases, respectively. Next, we chose shocks arising at different time points to describe the time-varying impulse response: 2008Q4, 2014Q2, and 2020Q1, representing the three periods of the global financial crisis, the international oil price crash, and COVID-19, respectively.

\subsection{The Effect of BDI on Stock Prices and GDP in China}

5.1.1. The Effect of Impulse Responses from Shocks at Three Different Periods Ahead. The impulse response of GDP to the BDI showed time-varying characteristics, as shown in Figure 2(a). The direction of the impulse response function 


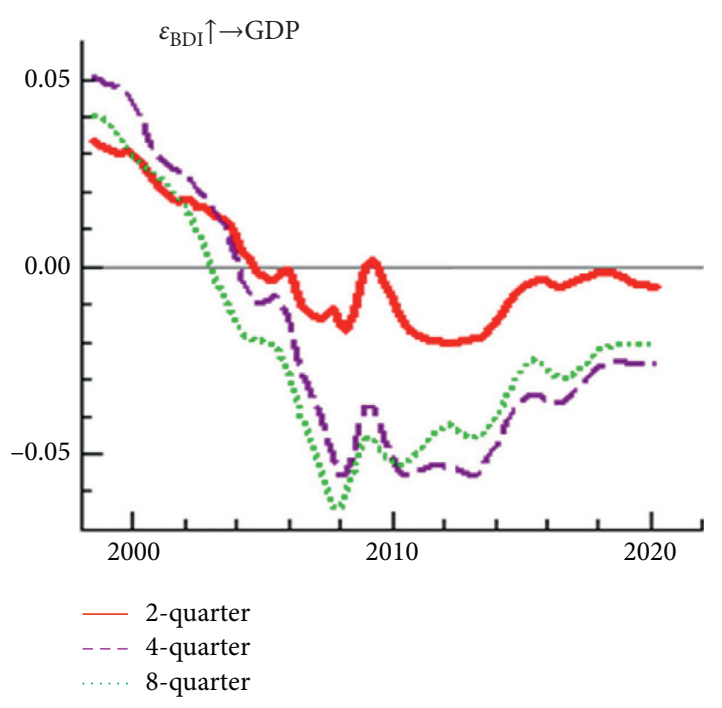

(a)

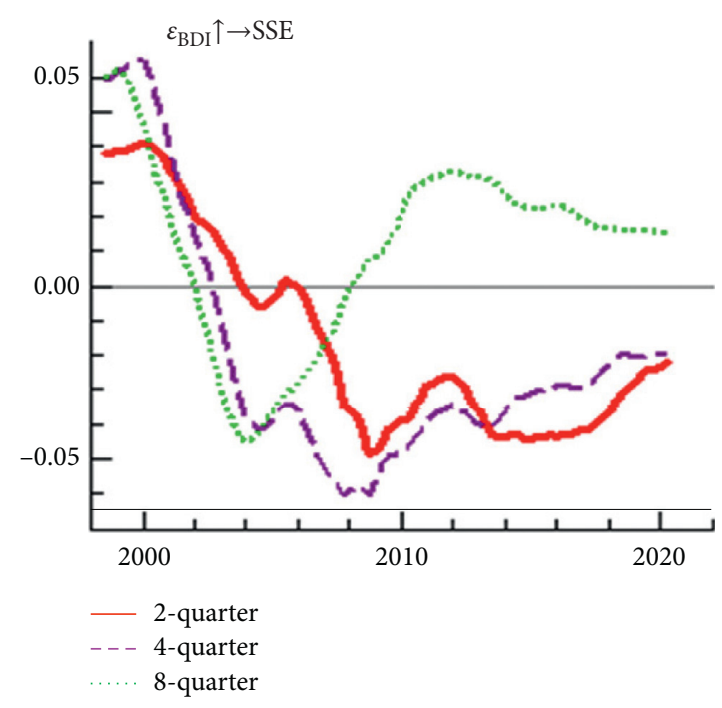

(b)

FIGURE 2: Impulse responses from shocks at three different periods ahead: (a) BDI $\longrightarrow$ GDP; (b) BDI $\longrightarrow$ SSE.

under different lag periods was basically the same. Specifically, it can be divided into three stages. The first stage was from 1998 to 2007. In this period, the impulse response of GDP to the BDI varied widely. The second stage was from 2008 to 2014, when the impact of the BDI on GDP was relatively smooth. The third stage was after 2015, when the impulse response of GDP to the BDI began to reverse, and the fluctuations became obvious. For the shock direction and intensity, in the first stage, there was a shift from "positive" to "negative" shocks. This was mainly due to the gradual increase in trade after the Asian financial crisis in 1997, when the economies of all countries began to recover. And, with China's accession to the WTO, China had become the world's number one processing plant with its low cost and promising market. China's economy began to boom. In this context, due to the economic recovery and the huge trade volume, the freight rates in the shipping market remained high, but the negative impact brought by the rise in shipping freight rates did not offset the positive impact brought by China's accession to the WTO on the economic interests of China. In other words, the positive impact of the increase in "trade volume" on China's economy was far greater than the negative impact of the increase in "freight rate" on China's economy. After that, during the period of 2004-2007, under the multiple drivers of the continuous growth of oil demand, frequent emergencies, and speculation, the international oil price began to enter the rising channel. Driven by the recovery of international trade demand and soaring oil prices, the BDI also showed favorable fluctuations and began to rise sharply, with the highest point breaking through 10,000 points, hitting a new historical high. After China's accession to the WTO, the volume of trade was huge, and the soaring BDI undoubtedly formed a constraint on the development of China's outbound-oriented economy. That is to mention, the negative impact of the increase in "freight prices" on China's economy outweighed the positive impact of the increase in "trade volume" on China's economy. In the second stage, the BDI produced negative shocks to GDP, with a smooth impulse response and the strongest shocks. After the 2008 financial crisis, the international oil price and the BDI plummeted. At this stage, GDP had the greatest response to the BDI shocks, indicating that the impact of the BDI on GDP was more pronounced during the period of increasing uncertainty in the international political and economic situations. In the third stage, the impulse response trend of GDP to the BDI began to reverse and to climb upward. It still showed a negative correlation, but the intensity of the shock gradually decreased. The impact of rising freight rates on China's economy stepped into a downward channel, which may be related to China's entry into the new normal of economic development and the transformation of the economic structure. The nonlinear dynamic response trends converged from different lag periods, but the lag 8 periods had the smallest shock strength, while the lag 2 and lag 4 periods' shocks were the strongest. As the lag period continues to increase, the impact intensity of the BDI to GDP gradually decreased.

The impact of the BDI on the SSE Composite Index was time-varying (BDI $\longrightarrow$ SSE), as shown in Figure 2(b). Similar to the GDP response, the stock price response to the BDI shock also showed a general trend from "positive" to "negative," but the difference was that the lag 8 period turned to positive after a short period of negative range fluctuation. For the perspective of different periods, the BDI mainly produced negative impact on stock price under lag 2 and lag 4 periods. For lag 8 period, there was a shift from "positive" to "negative" to "positive" impact on stock price, but it mainly showed positive impact. The results indicated that positive BDI shocks had a dampening effect on stock prices in the short and medium term, and the positive BDI shocks promoted stock market prosperity in the long-term perspective. Rising ship freight rates may make shipping stocks perform strongly, and there was a structural bull market. However, rising transportation costs for bulk commodity 
trade raised the cost of imports, which may have affected corporate performance. Thus, in the short and medium term, rising BDI dampened the stock market boom. From a long-term perspective, the continuous rise of BDI signaled the recovery and prosperity of the global economy, which was conducive to the formation of stable economic growth expectations for investors, further promoting stock market prosperity. Similar to GDP, it can also be divided into three stages: 1998-2007 (the impulse response function changed greatly and was unstable), 2008-2014 (the impulse response function fluctuated smoothly), and after 2015 (the impulse response function started to climb). For the shock direction and intensity, in the first stage, the impulse function under different lag periods were successively from "positive" to "negative." In the second stage, the lag 2 and 4 period impulse curves were less amplitude and basically stable, but the impact intensity was the strongest, while the lag 8 period impulse curve began to reverse, from "negative" to "positive." In the third stage, whether in the short, medium, or long term, the intensity of BDI shocks gradually converged.

\subsubsection{The Effect of Impulse Responses from Shocks at Three} Different Time Points. Figure 3 reflects the potential impact of BDI on Chinese stock market and economic growth at three different time points. The impulse response of GDP to the BDI (Figure 3(a)) depicted that the direction of shocks at three time points, 2018Q4, 2014Q2, and 2020Q1, were consistent and largely negative. However, the shocks varied in intensity at different time points, with the largest shock under the financial crisis, followed by the oil price crash, and the smallest shock under COVID-19. It indicated that the impact of China's economy on external shipping market shocks gradually decreased over time; that is, higher freight rates had a lower negative impact on the Chinese economy, which may be related to the gradual decrease in the dependence of the Chinese economy on foreign trade. From the impulse response of SSE to the BDI (Figure 3(b)), we found that the impulse response at different time points showed a trend from "positive" to "negative" to "positive." There was a negative impact in the short and medium term, but the long-term promotion effect was obvious, which further verified the previous analysis. For impact intensity, it also showed that the shock weakened over time and the ability to resist risks increased.

\subsection{The Effect of Brent on BDI, Chinese Stock Prices, and GDP}

5.2.1. The Effect of Impulse Responses from Shocks at Three Different Periods Ahead. According to the impulse response of GDP to Brent at three different periods ahead, the impulse response had significant nonlinear time-varying characteristics, which largely depended on the Chinese stage of economic development such as industrial structure optimization and business cycle fluctuations (Figure 4(a)). The impulse response of GDP to Brent was mainly divided into three stages, which were from 1998 to 2003 as the first stage, from 2004 to 2012 as the second stage, and from 2012 to 2020 as the third stage. In the first stage, the impulse response intensity from the short term, medium term, and long term was small. According to China's national conditions, this paper considered that China was building the basic framework of the socialist market economy system and was in the initial stage. In this stage, the impact of the international crude oil market had limited impact on Chinese economy. In the second stage, the impulse response intensity of GDP to Brent from the short term, medium term, and long term was more severe. There are two main reasons: the first point was that basic conditions for Chinese economic response to the international crude oil market have matured during the initial improvement of the socialist market economic system, such as the exchange rate system; the second point is that the rapid development of Chinese heavy chemical industry in this stage indirectly drove the demand for crude oil. However, due to the limited element endowments of crude oil, China was highly dependent on international crude oil imports, which further intensified the response of GDP to Brent. In the third stage, the impulse response intensity from the short term was still intense; however, the impulse response intensity from the mediumterm and long-term shocks gradually weakened. Based on Chinese development characteristics, we found that China had entered a stage of comprehensively deepening reform and development since 2012, in which the optimization of the industrial structure and economic development entered a new normal. Besides, the tertiary industry had the rapid development, and the proportion of energy-intensive industries has declined in this stage. The response of different industries to crude oil prices was heterogeneous in China, among which the metal mining and processing industries that used crude oil and its related products as power resources had the greatest negative effects. Therefore, the impulse response of GDP to Brent was blocked because of industrial optimization and structural transformation to a certain extent.

From the impact direction, the short-term impulse response function of Brent to GDP was positive. In terms of the direction of shocks, the short-term impulse response function of Brent to GDP was positive. It showed that there was a strong positive correlation between oil prices and China's economic growth, and higher oil prices stimulated economic growth in the short run. China was in a medium to high stage of development, with rapid industrial development and a strong demand for oil. When world oil prices rose, there was a time lag in decision-making in the industrial sector. Therefore, in the short term, rising oil prices boosted economic growth. In the medium and long term, oil prices affected economic development by influencing the cost of upstream production of raw materials to affect the downstream related production sector of factor inputs and the consumption side of the product price level and ultimately affected economic development. As a result, over time, the industrial sector became aware of the increased production costs associated with higher oil prices. Companies started taking practical actions and reduced their future production plans. Therefore, the existence of higher oil prices in the medium and long term had a negative impact on the economy. However, the impulse response of 


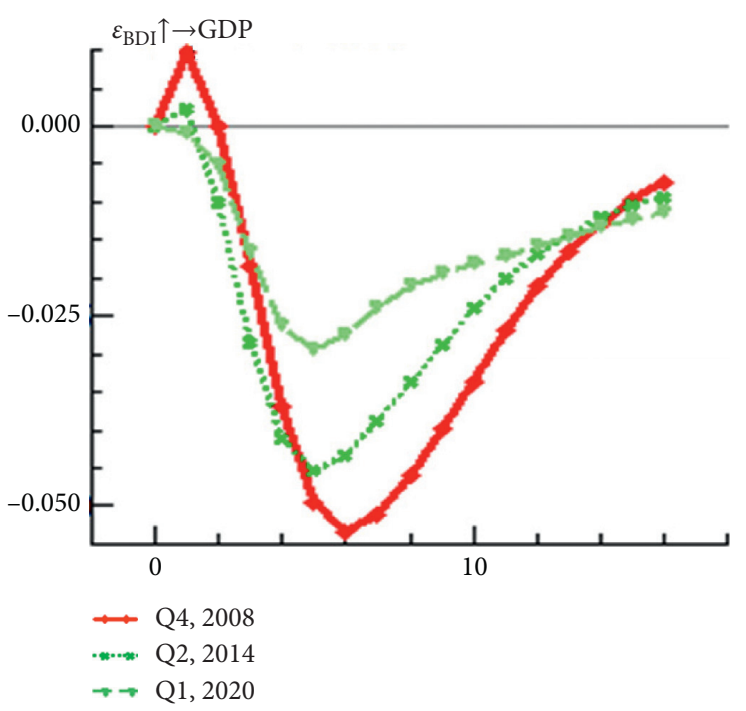

(a)

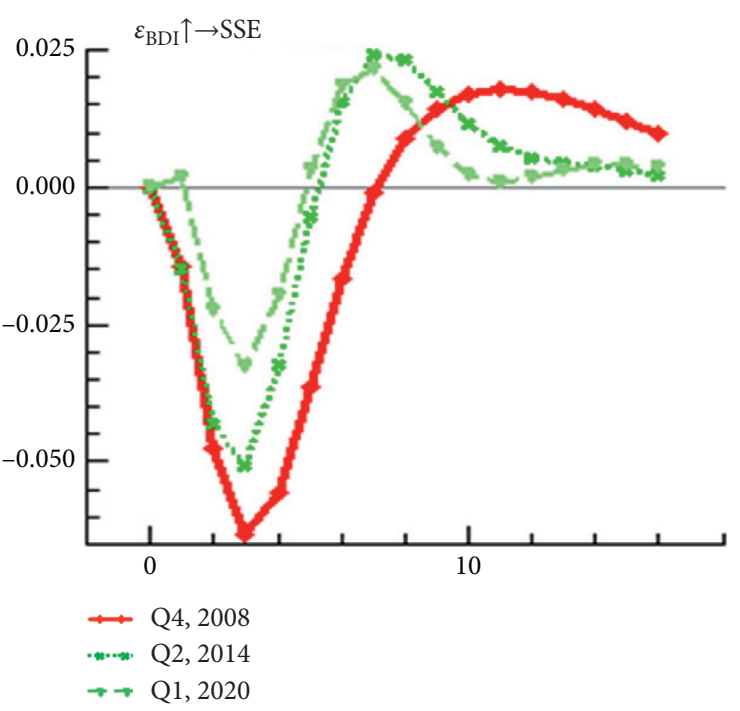

(b)

Figure 3: Impulse responses from shocks at three different time points: (a) BDI $\longrightarrow$ GDP; (b) BDI $\longrightarrow$ SSE.

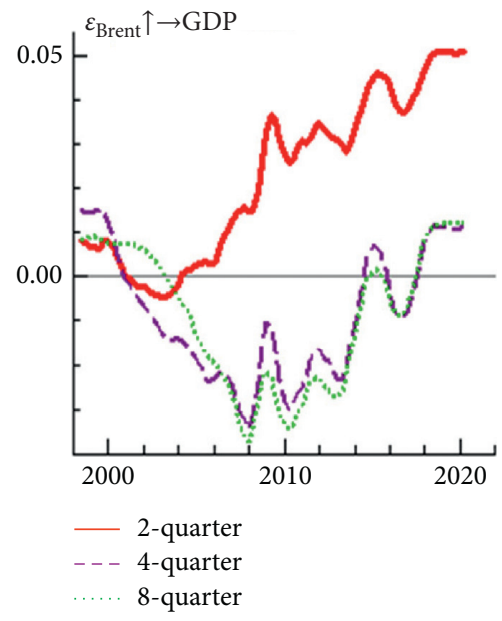

(a)

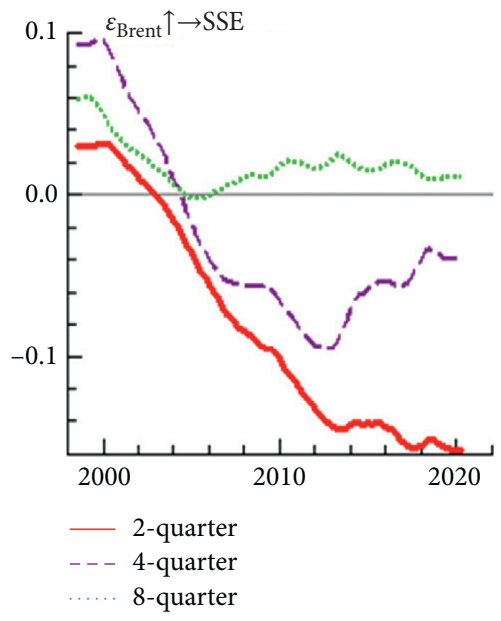

(b)

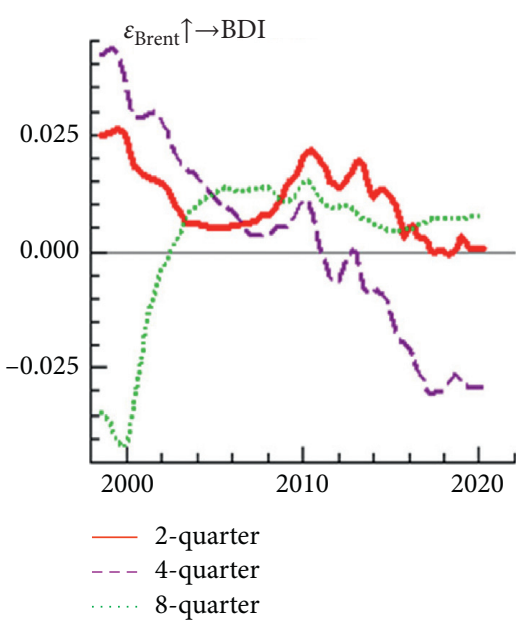

(c)

Figure 4: Impulse responses from shocks at three different periods ahead: (a) Brent $\longrightarrow$ GDP; (b) Brent $\longrightarrow$ SSE; (c) Brent $\longrightarrow$ BDI.

GDP to Brent turned from negative to positive. Oil price growth had a positive relationship with economic growth in this stage, which verified the research results of Du et al. [32] and Mo et al. [33].

The impulse response of SSE to Brent had obvious nonlinear characteristics (Figure 4(b)). The oil price affected the stock market in two main aspects. On the one hand, oil prices increased the cost of production, which affected corporate performance and ultimately stock prices. On the other side, it was transmitted through the intermediary variable of sentiment. The stronger the investor panic was, the greater the awareness of short selling was, which negatively affected stock prices. The first phase was from 1998 to 2005. The short-, medium-, and long-term impulse responses were positive, and the impact intensity gradually reduced. The stock market was in a growth period with a strong speculative atmosphere, which combined with the low oil price formed a positive stock market response to the crude oil market shock. The second stage was after 2006, when China achieved full liquidity of the stock market, and the stock market reform was basically completed. At this stage, the impulse response function was negative in the short and medium term but positive in the long term. In this paper, it was argued that the positive oil price shock deeply affected the production costs and investor sentiment of the whole industrial chain and thus played a restraining role on the stock market. So, the impulse response function in the short and medium term was consistent with economic theory. However, in the long run, Chinese economy had maintained a medium-to-high-speed development all year round, and oil prices had risen simultaneously with Chinese economic growth. Therefore, the long-term impulse response function conformed to the actual situation in China.

The impulse response of the BDI to Brent can be divided into two phases (Figure 4(c)). The first phase was from 1998 to 2004. In this phase, the impulse response of BDI to Brent 
was positive in the short and medium term, but negative in the long term. In the short term, positive fluctuations in crude oil prices were transmitted through transportation costs to the BDI, causing freight rates to rise, which was consistent with the short- and medium-term impulse response trends at this stage. But in the long run, the shipping market was more depressed at this stage, and rising oil prices played a restraining role on the shipping market. The second stage was after 2005. In this stage, the impulse response of the BDI to Brent was positive in the short term, which was consistent with economic logic. In the medium term, the impulse response of the BDI to Brent had a negative impact. In the long term, the impact was again positive. This may be due to the fact that the characteristics of oil as a commodity gradually emerged, and it rapidly rose due to speculation, unexpected events, and other factors, but the demand for iron ore, grain, and other dry bulk transport was relatively stable. Therefore, in the medium term, oil prices were running counter to the BDI phenomenon.

5.2.2. The Effect of Impulse Responses from Shocks at Three Different Time Points. The impact intensity of Brent on GDP at different time points showed heterogeneity (Figure 5(a)). From the impact direction, the impulse response of GDP to Brent shifted from "positive" to "negative" in period 6 during the 2008 financial crisis and the 2014 oil price crash. However, during the COVID-19 period, the impulse response of GDP to Brent always remained positive. From the impact intensity, the oil price shock to the Chinese economy during the 2008 financial crisis was the strongest and most harmful. Furthermore, the intensity impact of the oil price on China's economy in case of unexpected events gradually diminished over time, and the intensity impact of the oil price on China's economy even turned positive during COVID-19. This may be related to China's structural economic transformation and environmental policies such as "oil to gas."

As economic globalization continued to advance, the connection between markets was becoming closer, and the interaction between the international crude oil market and the Chinese stock market was strengthening, which was reflected in a more intense impulse response of the stock market to oil prices. Specifically, the impulse response trend of SSE to Brent at different time points was similar (Figure 5(b)). The impulse response of the stock market to Brent was predominantly negative. The impact intensity was the highest when the lag was two periods, and then, it gradually changed from negative to positive and finally converged to 0 . The results presented that the impact intensities at different time points were heterogeneous. Brent's impact intensity on the SSE was the highest during COVID19 and the lowest during the financial crisis. The impact intensity of Brent on the stock market was stronger during COVID-19 and the 2014 oil price crash than the impact intensity of Brent on the stock market during the $2008 \mathrm{fi}-$ nancial crisis. In addition, as China's stock market continued to open up and develop over time, the stock market had become more susceptible to investor sentiment and economic uncertainty and had become more responsive to major events. So, the impact intensity of oil price on the stock market increased.

The impulse response of the BDI to Brent exhibited distinct time-varying characteristics (Figure 5(c)). The impulse response of the BDI to Brent reached a maximum in the second period and then gradually weakened. The results showed that in the short run, the impact intensity of Brent on the BDI during the oil price crash and financial crisis was less than that of the COVID-19 period, and impact intensity decayed to zero after the $14^{\text {th }}$ period. During the 2008 financial crisis, the impulse response of the BDI to Brent was consistently positive, and the duration of Brent to BDI shocks was longer.

\subsection{The Dynamic Nexus between GDP and the Stock Market in China}

5.3.1. The Effect of Impulse Responses from Shocks at Three Different Periods Ahead. According to Chinese empirical data, the impulse response of GDP to SSE had a significant promotion effect (Figure 6(b)) at three different periods ahead. From impact intensity, a prosperous stock market boosted a prosperous economy. From the impulse response of SSE to GDP (Figure 6(a)), the Chinese stock market and the economic growth showed a positive correlation at three different periods ahead before 2008. After 2008, it began to reverse and showed a negative effect. This may be related to the successive introduction of countercyclical economic policies after the financial crisis, which led investors to gradually build up an expectation that the authorities would implement austerity policies when the economy overheats. In summary, there was a negative feedback adjustment mechanism between the stock market and the economy in China after 2008.

5.3.2. The Effect of Impulse Responses from Shocks at Three Different Time Points. Figure 7(b) demonstrates that the impulse response of GDP to SSE was positive at three different time points. Figure 7(a) demonstrates that the impulse response of SSE to GDP at three different time points was negative. It was further verified that there was a negative feedback adjustment mechanism between the Chinese economy and the stock market after 2008. And, the absolute value of the impact intensity of GDP on the stock market at different time points was greater than the absolute value of the impact intensity of SSE on GDP. In other words, the impact of the economy on the stock market was greater than the impact of the stock market on the economy.

5.4. Variance Decomposition of SSE and GDP. Table 3 shows the variance contribution of influencing factors to China's stock market. It can be seen from Table 3 that the main reason for the change of China's stock market was Brent, which had a greater impact on the stock market than BDI. Overall, the impact of the stock market on itself gradually decreased over time. First, GDP had little effect on the stock 


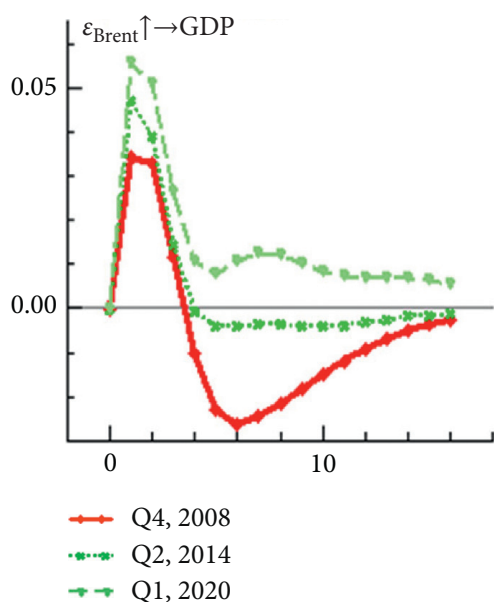

(a)

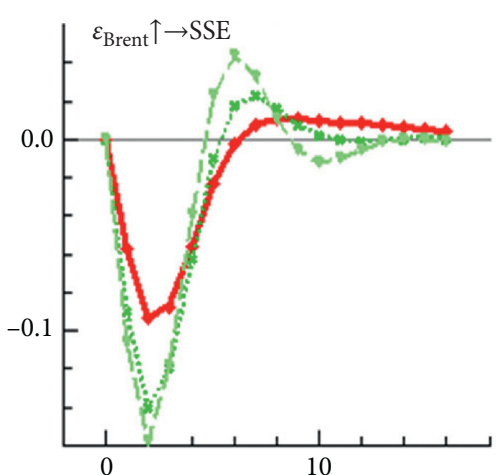

$\rightarrow$ Q4, 2008

..... Q2, 2014

$\rightarrow$ Q1, 2020

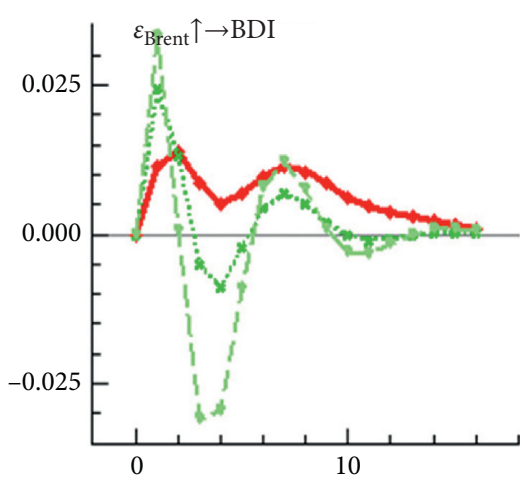

$\longrightarrow$ Q4, 2008

Q.-. Q2, 2014

$\rightarrow$ Q1, 2020

(c)

FIgure 5: Impulse responses from shocks at three different time points: (a) Brent $\longrightarrow$ GDP; (b) Brent $\longrightarrow$ SSE; (c) Brent $\longrightarrow$ BDI.

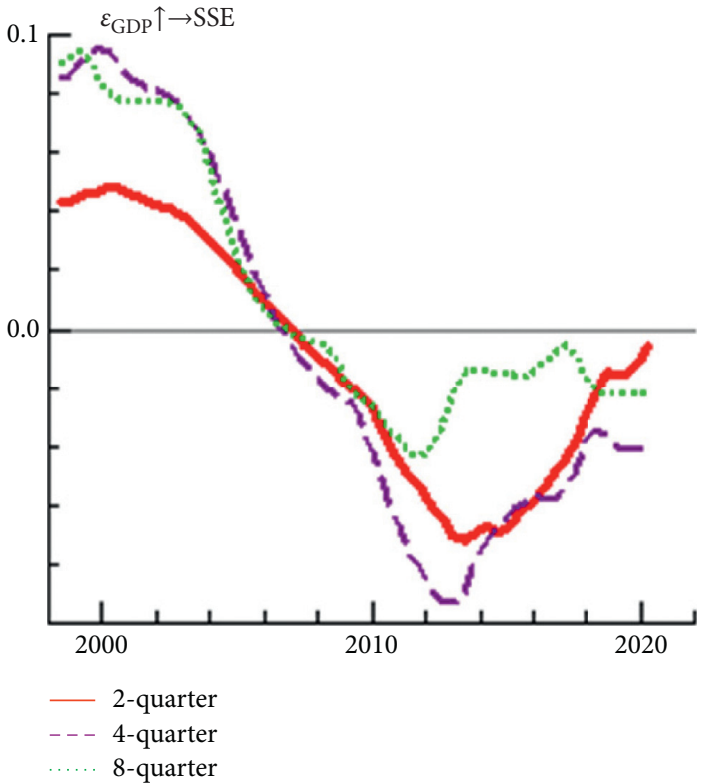

(a)

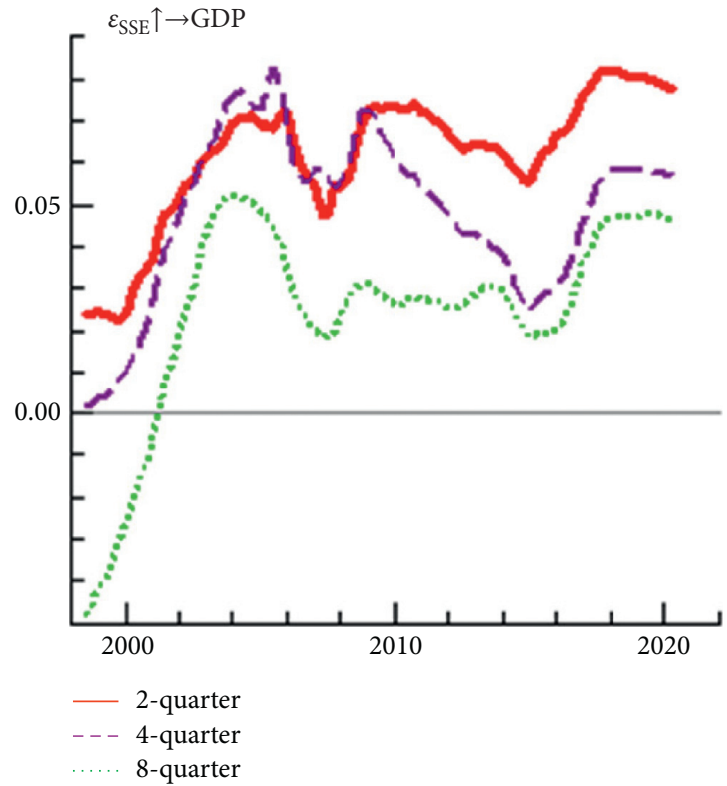

(b)

FIGURE 6: Impulse responses from shocks at three different periods ahead: (a) GDP $\longrightarrow$ SSE; (b) SSE $\longrightarrow$ GDP.

market in the current GDP. As the stock market is a barometer of the macro economy, GDP in the current period acted on the stock market by influencing investors' expectations. Therefore, the stock market was more influenced by investors' expectations of GDP. With the continuous extension of GDP lag period, investor expectations were constantly strengthened, and the impact on the stock market was also increasing. Second, Brent's impact on stocks has deepened over time. As oil is known as the blood of modern industry, the industrial sector is highly dependent on oil. As a traditional manufacturing country, China's energy external dependence is high, and the fluctuation of oil price has a profound influence on China's economy. The fluctuation of oil price affects the performance of listed companies and investors' economic expectations, thus causing stock market turbulence. Last, the contribution rate of BDI reached its peak in the third phase. Since the BDI mainly measures dry bulk shipments, they have less impact on the economy than oil. Therefore, the impact of the BDI on the stock market decreased slowly with the extension of the lag period.

Table 4 shows the variance contribution of GDP influencing factors. As can be seen from Table 4, the main influencing factor of GDP change was itself. With the continuous extension of the lag period, the contribution rate gradually decreased from $88.20 \%$ to $73.40 \%$. In terms of external factors, Brent's contribution to the Chinese economy was the highest, at around $20 \%$. As the lag period goes on, the contribution of the stock market and BDI to China's 


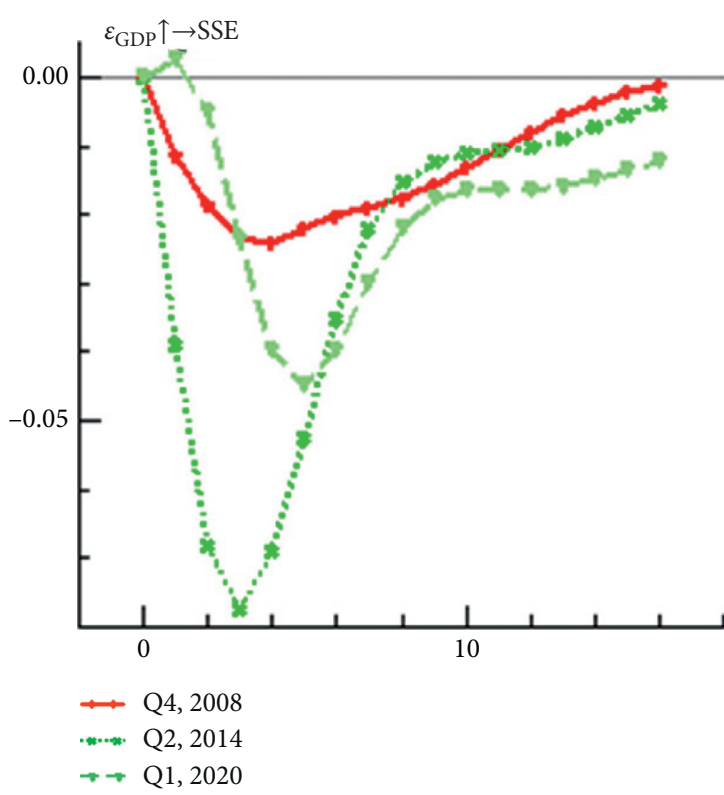

(a)

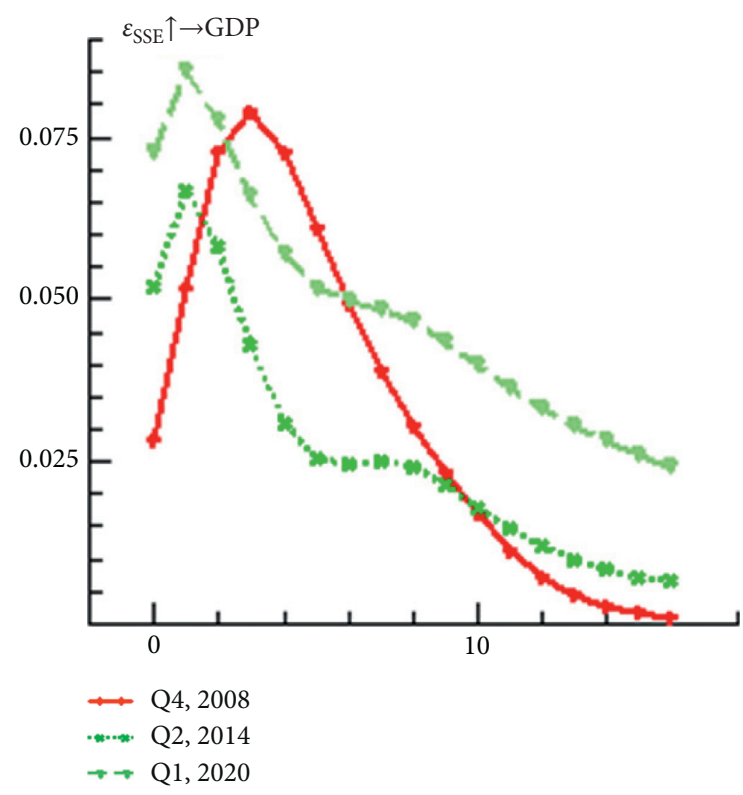

(b)

Figure 7: Impulse responses from shocks at three different time points: (a) GDP $\longrightarrow$ SSE; (b) SSE $\longrightarrow$ GDP.

TABLE 3: Variance decomposition of SSE.

\begin{tabular}{lcccc}
\hline Period & SSE & GDP & Brent & BDI \\
\hline 1 & 92.0898 & 0.0000 & 2.8734 & 5.0368 \\
2 & 86.3341 & 0.3192 & 2.0115 & 11.3352 \\
3 & 81.7636 & 0.7420 & 6.8049 & 10.6895 \\
4 & 77.2484 & 2.6126 & 10.6633 & 9.4758 \\
5 & 73.8604 & 5.3409 & 11.8618 & 8.9369 \\
6 & 72.2636 & 7.2789 & 11.6972 & 8.7603 \\
7 & 71.6974 & 7.8701 & 11.8249 & 8.6076 \\
8 & 71.2704 & 7.7403 & 12.5623 & 8.4270 \\
9 & 70.7282 & 7.6774 & 13.3012 & 8.2912 \\
10 & 70.2546 & 7.8582 & 13.6581 & 8.2291 \\
\hline
\end{tabular}

TABLE 4: Variance decomposition of GDP.

\begin{tabular}{lcccc}
\hline Period & GDP & SSE & Brent & BDI \\
\hline 1 & 88.1979 & 0.2228 & 11.5774 & 0.0019 \\
2 & 80.8341 & 0.3045 & 18.4991 & 0.3623 \\
3 & 77.3571 & 0.8465 & 21.4826 & 0.3138 \\
4 & 75.5825 & 1.4968 & 22.5697 & 0.3510 \\
5 & 74.8401 & 2.0422 & 22.5425 & 0.5752 \\
6 & 74.4040 & 2.2692 & 22.4427 & 0.8841 \\
7 & 74.1303 & 2.2880 & 22.3733 & 1.2084 \\
8 & 73.8841 & 2.2835 & 22.3617 & 1.4707 \\
9 & 73.6276 & 2.3354 & 22.3798 & 1.6572 \\
10 & 73.4008 & 2.4142 & 22.4091 & 1.7760 \\
\hline
\end{tabular}

economy gradually increased, but their contributions were generally low, which further demonstrated the importance of oil to manufacturing-oriented China.

\section{Conclusions}

This paper applied the TVP-SV-VAR model to analyze the nonlinear and time-varying effects of the world oil market and global shipping market on stock market and economic growth in China. The study used quarterly frequency data for the 1998Q1-2020Q2 period. The empirical results provide strong support for the presence of time-varying characteristics between the variables under study as follows:

First, from the impact direction, the impacts of the international shipping market on China's economy in different lag periods show a "positive" to "negative" shift. This is mainly due to the gradual increase in trade after the Asian financial crisis in 1997, when the economies of various countries begin to recover. And, with China's accession to the WTO, China has become the world's number one processing plant with its low cost and promising market. Against this backdrop, the negative impact of rising shipping costs cannot offset the positive impact of WTO accession on China's economic interests; that is, the positive impact of the increase in trade volume on China's economy is far greater than the negative impact of the increase in freight prices on China's economy. Then, as trade growth declines, BDI fluctuations begin to have a negative impact on the Chinese economy; that is, the negative impact of higher freight prices on China's economy outweighs the positive impact of higher trade volumes on China's economy. From the impact intensity, the impact of the BDI on China's economy and stock market is more pronounced during the period of increased uncertainty in the international political and economic 
situations. From the lag period, the impact intensity of the BDI on GDP has a distinct medium- to long-term effect as the lag period increases and the intensity of the shock increases. In addition, a positive BDI shock has a dampening effect on stock prices in the short and medium term, while a positive BDI shock can promote stock market prosperity in the long-term perspective. The impulse responses of SSE and GDP to BDI show that the external shipping market shocks to Chinese stock market and economic growth gradually become smaller over time, indicating that the ability to resist risks increases in China.

Second, the impulse response of China's economy and securities market to the fluctuation of international oil prices is affected by China's economic development, energy structure, major international emergencies, and the level of oil prices, which has a nonlinear time-varying characteristic. From the impact direction, the oil price has a strong positive correlation with China's economic growth due to the time lag in decision-making in the industrial sector, and rising oil prices can stimulate economic growth in the short-term. Over time, the industrial sectors gradually realize the problem of higher production costs caused by higher oil prices, and companies start to take practical actions and reduce their future production plans. In the medium to long term, therefore, the existence of higher oil prices has a negative impact on the economy. Furthermore, the intensity impact of the oil price on China's economy in case of unexpected events has gradually diminished over time, and the intensity impact of the oil price on China's economy even turned positive during COVID-19. This may be related to China's structural economic transformation and environmental policies such as the "oil to gas." In addition, as China's stock market continues to open up and develop over time, the stock market has become more susceptible to investor sentiment and economic uncertainty and has become more responsive to major events. So, the impact intensity of oil price on the stock market has increased.

Last, after the 2008 financial crisis, the stock market boom has promoted economic prosperity, and economic prosperity has dampened stock market boom conversely, which proves that there existed a negative feedback adjustment mechanism between the stock market and the economy. Besides, the absolute value of the impact intensity of GDP on the stock market at different time points is greater than the absolute value of the impact intensity of the stock market on GDP; i.e., the economy affects the stock market more than the stock market affects the economy.

\section{Data Availability}

All data used have been included in the Wind database and are also available from the corresponding author upon request.

\section{Conflicts of Interest}

The authors declare that there are no conflicts of interest regarding the publication of this paper.

\section{Acknowledgments}

This work was supported by the National Natural Science Foundation of China (NSFC) (71874133), Annual Basic Scientific Research Project of Xidian University (2019), and Youth Innovation Team of Shanxi Universities.

\section{References}

[1] X. Zhang, T. Xue, and H. E. Stanley, "Comparison of econometric models and artificial neural networks algorithms for the prediction of baltic dry index," IEEE Access, vol. 1, 2018.

[2] R. Qingsong, W. Yao, and L. Xinsheng, "Cross-correlations between baltic dry index and crude oil prices," Physica A: Statistical Mechanics and its Applications, vol. 453, 2016.

[3] A. Arigoni, A. Newman, C. Turner, and C. Kaptur, "Optimizing global thermal coal shipments," Omega, pp. 118-127, Elsevier, Amsterdam, Netherlands, 2017.

[4] P.-y. Nie and Y.-C. Yang, "Effects of energy price fluctuations on industries with energy inputs: an application to China," Applied Energy, vol. 165, pp. 329-334, 2016.

[5] B. Ou, X. Zhang, and S. Wang, "How does China's macroeconomy response to the world crude oil price shock: a structural dynamic factor model approach," Computers \& Industrial Engineering, vol. 63, no. 3, pp. 634-640, 2012.

[6] S. A. Basher, A. A. Haug, and P. Sadorsky, "Oil prices, exchange rates and emerging stock markets," Energy Economics, vol. 34, no. 1, pp. 227-240, 2012.

[7] D. Cheng, X. Shi, J. Yu, and D. Zhang, "How does the Chinese economy react to uncertainty in international crude oil prices?" International Review of Economics \& Finance, vol. 64, 2019.

[8] L. Zhao, X. Zhang, S. Wang, and S. Xu, "The effects of oil price shocks on output and inflation in China," Energy Economics, vol. 53, pp. 101-110, 2016.

[9] W. J. Kim, S. Hammoudeh, J. S. Hyun, and R. Gupta, "Oil price shocks and China's economy: reactions of the monetary policy to oil price shocks," Energy Economics, vol. 62, pp. 61-69, 2017.

[10] H. Bloch, S. Rafiq, and R. Salim, "Economic growth with coal, oil and renewable energy consumption in China: prospects for fuel substitution," Economic Modelling, vol. 44, pp. 104-115, 2015.

[11] T. Liang, J. Chai, Y.-J. Zhang, and Z. G. Zhang, "Refined analysis and prediction of natural gas consumption in China," Journal of Management Science and Engineering, vol. 4, no. 2, pp. 91-104, 2019.

[12] P. K. Narayan and S. S. Sharma, "New evidence on oil price and firm returns," Social Science Electronic Publishing, vol. 35, no. $12,2011$.

[13] P. K. Narayan and S. S. Sharma, "Firm return volatility and economic gains: the role of oil prices," Economic Modelling, vol. 38, pp. 142-151, 2014.

[14] D. H. B. Phan, S. S. Sharma, and P. K. Narayan, "Oil price and stock returns of consumers and producers of crude oil," Journal of International Financial Markets, Institutions and Money, vol. 34, pp. 245-262, 2015.

[15] D. H. B. Phan, S. S. Sharma, and P. K. Narayan, "Stock return forecasting: some new evidence," International Review of Financial Analysis, vol. 40, pp. 38-51, 2015.

[16] X. Wen, Y. Guo, Y. Wei, and D. Huang, "How do the stock prices of new energy and fossil fuel companies correlate? 
evidence from China," Energy Economics, vol. 41, pp. 63-75, 2014.

[17] Z. Ding, Z. Liu, Y. Zhang, and R. Long, "The contagion effect of international crude oil price fluctuations on Chinese stock market investor sentiment," Applied Energy, vol. 187, pp. 27-36, 2017.

[18] O. Erdogan, K. Tata, B. C. Karahasan et al., "Dynamics of the co-movement between stock and maritime markets," International Review of Economics \& Finance, vol. 25, 2013.

[19] A. H. Alizadeh and G. Muradoglu, "Stock market efficiency and international shipping-market information," Journal of International Financial Markets, Institutions and Money, vol. 33, pp. 445-461, 2014.

[20] A. J. Lin, H. Y. Chang, and J. L. Hsiao, "Does the baltic dry index drive volatility spillovers in the commodities, currency, or stock markets?" Transportation Research Part E: Logistics and Transportation Review, vol. 127, pp. 265-283, 2019.

[21] E. Melike, "Baltic dry index as a major economic policy indicator: the relationship with economic growth," ProcediaSocial and Behavioral Sciences, vol. 210, pp. 416-424, 2015.

[22] F. Lin and N. C. S. Sim, "Trade, income and the baltic dry index," European Economic Review, vol. 59, pp. 1-18, 2013.

[23] F. Lin and D. Fu, "Trade, institution quality and income inequality," World Development, vol. 77, pp. 129-142, 2016.

[24] L. Han, J. Jin, L. Wu, and H. Zeng, "The volatility linkage between energy and agricultural futures markets with external shocks," International Review of Financial Analysis, vol. 68, 2020.

[25] L. Han, Li Wan, and Y. Xu, "Can the baltic dry index predict foreign exchange rates?" Finance Research Letters, vol. 32, 2020.

[26] Y.-J. Zhang, "Speculative trading and WTI crude oil futures price movement: an empirical analysis," Applied Energy, vol. 107, pp. 394-402, 2013.

[27] D. Zhang, Q. Ji, and A. M. Kutan, "Dynamic transmission mechanisms in global crude oil prices: estimation and implications," Energy, vol. 175, pp. 1181-1193, 2019.

[28] S. J. Byun, "Speculation in commodity futures markets, inventories and the price of crude oil," The Energy Journal, vol. 38, no. 5, pp. 93-113, 2017.

[29] E. D. Attanasi, "Bitumen prices and structural changes in north American crude oil markets," Natural Resources Research, vol. 25, no. 4, pp. 1-10, 2016.

[30] L. Tingting, L. Chao, and S. Rui, "The structure and dynamics of granular complex networks deriving from financial time series," International Journal of Modern Physics C (IJMPC), vol. 31, 2020.

[31] H. Ding, H.-G. Kim, and S. Y. Park, "Crude oil and stock markets: causal relationships in tails?" Energy Economics, vol. 59, pp. 58-69, 2016.

[32] L. Du, H. Yanan, and C. Wei, “The relationship between oil price shocks and China's macro-economy: an empirical analysis," Energy Policy, vol. 38, no. 8, pp. 4142-4151, 2010.

[33] B. Mo, C. Chen, H. Nie, and Y. Jiang, "Visiting effects of crude oil price on economic growth in BRICS countries: fresh evidence from wavelet-based quantile-on-quantile tests," Energy, vol. 178, no. 1, pp. 234-251, 2019. 